\title{
A Cognitive Behavioral Therapy Group for Adolescent and Young Adult Cancer Patients: A Review of a Pilot Program
}

\author{
Brittany C. Hall, PhD, ${ }^{1-3}$ Victoria M. Short, ${ }^{2,4}$ Scott Giberson, ${ }^{2,4}$ and Laura Howe-Martin, $\mathrm{PhD}^{1,2}$
}

Adolescent and young adult (AYA-between ages 15 and 39) cancer patients face unique psychosocial challenges due to their developmental stage and complex health problems. Research indicates psychotherapy and group support can be beneficial to AYAs. Group therapy offers an opportunity for connection among peers who are enduring similar struggles while also utilizing effective therapeutic intervention. The current project describes a model for an AYA cognitive behavioral therapy (CBT) group. The model was informed by existing CBT literature and was successfully implemented. Future research may evaluate the efficacy of the proposed group structure and content for reducing distress in AYA cancer patients.

Keywords: group CBT, cognitive behavior therapy group, CBT group structure, AYA, oncology

\section{Introduction}

A DOLESCENT AND YOUNG ADULT (AYA) cancer patients are in a developmentally unique stage of life. AYAs, defined here as individuals aged 15 to 39 , are transitioning from childhood - a stage of life characterized by dependence on others - to the autonomy of adulthood, and a cancer diagnosis can significantly complicate this phase of development. AYAs present with higher levels of anxiety and depression, compared to both younger children and older adults with cancer. ${ }^{1,2}$ In addition, AYAs with cancer may be inhibited from participating in pivotal or daily routine activities. ${ }^{3}$ This, in turn, widens the gap of experiences and commonalities between individuals with and without cancer, and may further discourage AYAs from building or maintaining relationships with same-aged peers. AYAs with cancer are also at specific risk for distress and isolation due to their diagnosis and decreased social functioning. ${ }^{4}$

Research has demonstrated that cancer patients with stronger support systems are better equipped to process and cope with cancer-related distress. ${ }^{5}$ In particular, AYAs favor peers as sources of support and desire social acceptance more than other age groups, even in the absence of a cancer diagnosis. ${ }^{6}$ Group interventions for cancer patients have been shown to be beneficial in offering peer guidance and support while reducing depressive symptoms; however, group therapy has received little attention in AYA cancer treatment. ${ }^{7}$ In the context of group therapy, the mental health care provider is not the primary or sole resource, as the patient has access to other individuals with similar experiences. Furthermore, group therapy for AYAs with cancer could increase accessibility of treatment and capitalize on available mental health resources.

Although research on the effectiveness of group therapy for AYA cancer patients is limited, research has shown that cognitive behavioral therapy (CBT) groups for older adult cancer patients are effective. A CBT group for women with nonmetastatic breast cancer led to reduced reports of emotional distress, anxiety, and cancer-related thought intrusions. $^{8}$ Researchers in Ireland investigating CBT group therapy with breast cancer patients found a reduction in global stress and anxiety, with a greater effect being present among individuals with higher baseline levels of global stress. ${ }^{9}$ Another CBT group for breast cancer patients found that participants experienced decreased severity and frequency of their menopausal symptoms as a result of targeted interventions, and consequently experienced improvements in depressed mood and anxiety. ${ }^{10}$ Based on the current research demonstrating the efficacy of group CBT in older adult cancer patients, group CBT for AYA cancer patients may warrant further exploration.

Research has demonstrated AYAs with cancer are receptive to group therapy, as they are able to meet individuals of a similar age who are experiencing similar problems. ${ }^{11}$ AYA online support groups have been a useful resource for exchanging support, coping, and becoming part of a community

${ }^{1}$ Department of Psychiatry, UT Southwestern Medical Center, Dallas, Texas, USA.

${ }^{2}$ UT Southwestern Moncrief Cancer Institute, Fort Worth, Texas, USA.

${ }^{3}$ Fort Worth Adolescent Young Adult Oncology Coalition, Fort Worth, Texas, USA.

${ }^{4}$ Department of Psychology, Texas Christian University, Fort Worth, Texas, USA. 
that faces unique struggles. ${ }^{12}$ Recently, a group of researchers in Australia facilitated televideo therapy groups for AYAs with cancer. ${ }^{13-15}$ The groups utilized a CBT approach, and results supported the acceptability and feasibility of the treatment protocol with this population. ${ }^{15}$ Further research of this group model found that participants regarded the group as highly beneficial and not burdensome. ${ }^{13}$ In addition, participants reported feeling accepted throughout the intervention, and their comfort with sharing in the group increased comparably from the first to last session. ${ }^{13}$ In summary, there are unique risks of depression, anxiety, and isolation among AYA cancer patients and research highlights the potential benefits of AYA group therapy. Therefore, the current article identifies a structured CBT group intervention for AYAs with cancer and reviews the pilot implementation of this group within a clinical setting.

\section{Program Description}

\section{Participants}

We offered a 6-week CBT therapy group to AYA cancer survivors aged 18 to 39 . Group members were eligible to participate if they had a previous cancer diagnosis. No exclusions were made based on diagnosis or time since diagnosis. The therapy group was offered at an outpatient cancer institute that provides psychosocial and supportive services to adult cancer survivors. Four iterations of the 6-week group were delivered to AYAs with slight variations, with enrollment ranging from 2 to 6 participants per group. A total of 17 participants completed at least 1 group session, and 3 AYAs repeated participation in the 6-week group and attended more than 6 sessions. Of the remaining 14 AYAs, 3 attended 1 session, 4 attended 2 sessions, 3 attended 3 sessions, 2 attended 5 sessions, and 2 attended all 6 sessions.

AYAs were referred to the group from internal staff, such as social workers, nurse navigators, dietitians, genetic counselors, and exercise specialists, and also from local oncology clinics.
An AYA nurse navigator who provides navigation services within the county emailed flyers to previously identified patients in the community. Flyers advertising the group were also posted within the cancer institute. Potential group members could also self-refer.

\section{Group therapy providers}

Each 6-week group was consistently led by a licensed psychologist (B.C.H.) and a psychology doctoral intern. The psychology doctoral interns were provided supervision in AYA services, group therapy, and CBT.

\section{Intake process}

The majority of group members received an intake interview before participation in group to provide basic education on the group format, assess current mental health problems, and address potential concerns about participating in a therapy group. Not all group members completed the intake process as they missed their intake appointment; however, due to the timeline of the group start date, they were not excluded from participating. Group members were informed that they were expected to attend five out of the six sessions to be able to participate; no other exclusion criteria were established.

\section{CBT group structure and components}

The structure of the CBT group was informed by research specific to common problems cancer survivors and AYAs face. Interventions were chosen based on their efficacy and rationale in the literature. Each group utilized the typical CBT structure of setting an agenda, reviewing homework, teaching a new skill, eliciting feedback, and assigning new homework. Topics included behavioral activation, relaxation techniques, cognitive restructuring, components of CBT-insomnia, and management of medical care (refer to Table 1 for detailed group structure and rationale).

Table 1. Six-Week CBT Group Structure Outline

\begin{tabular}{|c|c|c|}
\hline Session & Content covered & Rationale/research support \\
\hline 1 & $\begin{array}{l}\text { Group guidelines } \\
\text { Personal cancer stories } \\
\text { Introduce CBT model } \\
\text { Introduce homework: emotion/behavior tracking }\end{array}$ & $\begin{array}{l}\text { Psychoeducation is often incorporated into effective } \\
\text { CBT interventions for cancer patients }{ }^{18} \text {; } \\
\text { psychoeducation groups overall have been found to } \\
\text { be more effective than support groups with cancer } \\
\text { survivors. }\end{array}$ \\
\hline 2 & $\begin{array}{l}\text { Homework review } \\
\text { New CBT skills: BA \& PMR } \\
\text { Introduce homework: daily activity diary }\end{array}$ & $\begin{array}{l}\text { BA is effective with breast cancer survivors }{ }^{20} \text {; PMR } \\
\text { for anxiety reduced fatigue and increased sleep } \\
\text { quality in breast cancer patients }\end{array}$ \\
\hline $3 \& 4$ & $\begin{array}{l}\text { Homework review } \\
\text { New CBT skill: cognitive distortions \& restructuring } \\
\text { Introduce homework: thought records }\end{array}$ & $\begin{array}{l}\text { CBT with cognitive restructuring reduced anxiety in } \\
\text { terminal cancer patients }{ }^{22}\end{array}$ \\
\hline 5 & $\begin{array}{l}\text { Homework review } \\
\text { New CBT skill: healthy sleep habits \& interventions } \\
\text { Introduce homework: sleep diary }\end{array}$ & $\begin{array}{l}\text { Sleep quality and fatigue is a significant problem } \\
\text { among AYAs with cancer }{ }^{23} \text {; while CBT for } \\
\text { insomnia is effective for cancer patients }\end{array}$ \\
\hline 6 & $\begin{array}{l}\text { Homework review } \\
\text { Identify and problem solve current challenges in } \\
\text { medical care } \\
\text { OR } \\
\text { Q \& A with expert AYA panel: AYA Oncologist, } \\
\text { Nurse Navigator, P.A., Genetic Counselor }\end{array}$ & $\begin{array}{l}\text { AYA cancer patients often report unmet psychosocial } \\
\text { and medical needs when treated in an adult cancer } \\
\text { facility }{ }^{25}\end{array}$ \\
\hline
\end{tabular}




\section{Conclusions}

\section{Applicability of CBT group therapy to AYAs}

All information obtained in the conclusions section was obtained through group leader observation and qualitative responses that group participants provided throughout group. Overall, our observations were that the proposed group outline provided good structure for group members to share personal experiences and learn new information. For example, group members "checked-in", with one another at the beginning of each session by reviewing their homework from the previous week. This model allowed for group cohesion and sharing of personal information in a structured way. During homework review, group members shared challenging situations from the previous week when they attempted to use the assigned skill. They discussed whether they used the skill and were successful; if they needed help, they would troubleshoot with other group members how to more effectively manage their problem.

In addition, interventions chosen were principle-driven in nature, thus, could be applied to a wide array of presenting problems. For example, group members were taught how to identify thoughts, emotions, behaviors, and physiological sensations, and they were instructed how to change behavior (behavioral activation), physiological sensations (progressive muscle relaxation), or thoughts (cognitive restructuring) to influence emotions. The skills were taught using common problematic thoughts that AYAs face, including thoughts related to cancer recurrence, appearance, or changes in independence. This approach was combined with more skillspecific interventions for sleep and management of medical care. Balancing a principle-based approach with problemspecific interventions not only addressed both the unique problems that AYAs face but also promoted generalization to more common mental health symptoms. Group member participation and engagement during sessions appeared high, potentially as a result of this model.

Although the CBT model allowed for practice of the newly learned skills between sessions, few group members routinely completed homework assignments between sessions. This is potentially problematic for an intervention-based group, as research indicates that completion of homework is directly related to increased improvements in outcomes. ${ }^{16}$ These concerns were not widely addressed in the structure of the outlined group due to time limitations; however, the importance of homework completion could be reviewed more extensively in an individual intake or orientation session before the initiation of group.

\section{Pros and cons to 6-week session and closed group format}

The 6-week structure of group allowed sufficient time to introduce and practice new interventions in group. Group members were provided with sufficient information to begin practicing new skills, gauge if additional support was needed, and be able to enroll in individual therapy services to continue associated intervention.

However, some important themes that group members repeatedly expressed interest in were not covered explicitly in group due to time limitations. These areas included difficulties in sexual functioning, physical appearance, and gen- eral health behaviors. While these topics were discussed in the larger framework of cognitive restructuring and behavioral activation, specific interventions and psychoeducation may have been able to be provided if the group had additional sessions.

Regarding the closed group format, group members were able to receive ongoing support from a similar group of peers, and this provided stability and the opportunity for group cohesion. However, multiple group members participated in multiple iterations of the group. While group members may benefit from reiteration of skills, there are limitations to repeat participation. For example, group repeaters may be less engaged and committed as there are new group members and much of the reviewed material is redundant. However, they may benefit from ongoing peer support; a solution for group member completers could be to attend a once monthly "graduate" support group or group booster session.

\section{The benefit of limiting exclusion criteria}

By not restricting group membership by age or stage of treatment, group members were able to naturally learn from one another and provide practical advice and support to group members who were more recently diagnosed or entering into a new stage of survivorship. Therefore, a mentorship model naturally developed; however, even the "mentors" continued to receive support through skill development within the group. This format differs from other existing programs where "mentors" may be matched with a more novice survivor. The current group implementation promoted ongoing learning to survivors at any stage of the journey. This is important as research has demonstrated that AYA cancer survivors who have transitioned in to survivorship report a desire to receive support from other AYA survivors. ${ }^{17}$ In addition, the current group did not make restrictons about type of diagnosis. There are limited numbers of AYAs in most clinics, and often there is an insufficient number of AYA referrals to create a disease-specific group or agerestricted group. Furthermore, group members learned about the variety of experiences of AYAs outside the scope of their specific diagnosis, which may have broadened their understanding of how peers are affected by cancer. Unless the group is psychoeducational in nature and designed for a specific disease process, the benefits of broadening the scope of referrals may outweigh making specifications that limit AYA patient inclusion.

\section{Future directions}

Limited research is available on the effectiveness of AYA group therapy to reduce psychological symptoms and cancerrelated distress. This implementation project examined common dilemmas clinicians face in creating and initiating a therapy group, and it proposed a structure for a CBT therapy group that was successfully and repeatedly implemented with AYAs. Future research should examine clinical outcomes of AYAs who participate in the 6-week group and also assess unmet needs of group member completers. These results could be utilized to identify appropriate interventions to incorporate into the existing structure. In addition, a formal clinical trial could examine outcomes comparing a CBTspecific group to a more general support group for AYAs or 
another form of group psychotherapy. This information has important implications for staff utilization within cancer treatment centers, particularly when bandwidth is limited. Furthermore, implementing effective methods for reducing psychiatric symptoms may reduce the burden of these symptoms to the patient, the patient's psychosocial system, and the medical community.

\section{Author Disclosure Statement}

No competing financial interests exist.

\section{Funding Information}

Authors B.C.H. and L.H. received partial salary support from the Fort Worth Adolescent and Young Adult Oncology Coalition, a non-profit entity.

\section{References}

1. Kessler RC, Chiu WT, Demler O, Walters EE. Prevalence, severity, and comorbidity of 12-month DSM-IV disorders in the national comorbidity survey replication. Arch Gen Psychiatry. 2005;62(6):617-27.

2. Lang MJ, David V, Giese-Davis J. The age conundrum: a scoping review of younger age or adolescent and young adult as a risk factor for clinical distress, depression, or anxiety in cancer. J Adolesc Young Adult Oncol. 2015; 4(4):157-73.

3. Bellizzi KM, Smith A, Schmidt S, et al. Positive and negative psychosocial impact of being diagnosed with cancer as an adolescent or young adult. Cancer. 2012;118(20): 5155-62.

4. Smith AW, Bellizzi KM, Keegan TH, et al. Health-related quality of life of adolescent and young adult patients with cancer in the United States: the adolescent and young adult health outcomes and patient experience study. J Clin Oncol. 2013;31(17):2136-45.

5. Usta YY. Importance of social support in cancer patients. Asian Pac J Cancer Prev. 2012;13(8):3569-72.

6. Zarrett N, Eccles J. The passage to adulthood: challenges of late adolescence. New Dir Youth Dev. 2006;2006(111): 13-28.

7. Faller H, Schuler M, Richard M, et al. Effects of psychooncologic interventions on emotional distress and quality of life in adult patients with cancer: systematic review and meta-analysis. J Clin Oncol. 2013;31(6):782-93.

8. Antoni MH, Wimberly SR, Lechner SC, et al. Reduction of cancer-specific thought intrusions and anxiety symptoms with a stress management intervention among women undergoing treatment for breast cancer. Am J Psychiatry. 2006;163(10):1791-7.

9. Groarke A, Curtis R, Kerin M. Cognitive-behavioural stress management enhances adjustment in women with breast cancer. Br J Health Psychol. 2013;18:623-41.

10. Hunter MS, Coventry S, Hamed H, et al. Evaluation of a group cognitive behavioral intervention for women suffering from menopausal symptoms following breast cancer treatment. Psychooncology. 2009;18(5):560-3.

11. Roberts CS, Piper L, Denny J, Cuddeback G. A support group intervention to facilitate young adults' adjustment to cancer. Health Soc Work. 1997;22(2):133-41.

12. Love B, Crook B, Thompson CM, et al. Exploring psychosocial support online: a content analysis of messages in an adolescent and young adult cancer community. Cyberpsychol Behav Soc Netw. 2012;15(10):555-9.

13. McGill BC, Sansom-Daly UM, Wakefield CE, et al. Therapeutic alliance and group cohesion in an online support program for adolescent and young adult cancer survivors: lessons from "Recapture Life." J Adolesc Young Adult Oncol. 2017;6(4):568-72.

14. Sansom-Daly UM, Wakefield CE, McGill BC, Patterson P. Ethical and clinical challenges delivering group-based cognitive-behavioural therapy to adolescents and young adults with cancer using videoconferencing technology. Aust Psychol. 2015;50(4):271-8.

15. Sansom-Daly UM, Wakefield CE, Bryant RA, et al. Feasibility, acceptability, and safety of the Recapture Life videoconferencing intervention for adolescent and young adult cancer survivors. Psychooncology. 2019; 28(2):284-92.

16. Mausbach BT, Moore R, Roesch S, et al. The relationship between homework compliance and therapy outcomes: an updated meta-analysis. Cognit Ther Res. 2010;34(5):429-38.

17. Millar B, Patterson P, Desille N. Emerging adulthood and cancer: how unmet needs vary with time-since-treatment. Palliat Support Care. 2010;8(2):151-8.

18. Dolbeault S, Cayrou S, Bredart A, et al. The effectiveness of a psycho-educational group after early-stage breast cancer treatment: results of a randomized French study. Psychooncology. 2009;18(6):647-56.

19. Edelman S, Craig A, Kidman AD. Group interventions with cancer patients: efficacy with cancer patients. J Psychosoc Oncol. 2008;18(3):67-85.

20. Ryba MM, Lejuez CW, Hopko DR. Behavioral activation for depressed breast cancer patients: the impact of therapeutic compliance and quantity of activities completed on symptom reduction. J Consult Clin Psychol. 2014;82(2):325-35.

21. Damiralp M, Oflaz F, Komurcu S. Effects of relaxation training on sleep quality and fatigue in patients with breast cancer undergoing adjuvant chemotherapy. J Clin Nurs. 2010;19(7-8):1073-83.

22. Greer JA, Traeger L, Bemis H, et al. A pilot randomized controlled trial of brief cognitive-behavioral therapy for anxiety in patients with terminal cancer. Oncologist. 2012; 17(10):1337-45.

23. Fortmann J, Fisher A, Hough R, et al. Sleep quality, fatigue, and quality of life among teenage and young adult cancer survivors. J Adolesc Young Adult Oncol. 2018;7(4):465-71.

24. Fleming L, Randell K, Harvey C, Espie CA. Does cognitive behavior therapy for insomnia reduce clinical levels of fatigue, anxiety, and depression in cancer patients? Psychooncology. 2014;23(6):679-84.

25. Zebrack BJ, Block R, Brandon H, et al. Psychosocial service use and unmet need among recently diagnosed adolescent and young adult cancer patients. Cancer. 2013; 119(1):201-14.

Address correspondence to: Brittany C. Hall, PhD Department of Psychiatry UT Southwestern Moncrief Cancer Institute 400 West Magnolia Avenue Fort Worth, TX 76104

USA

Email: brittanyc.hall@utsouthwestern.edu 